\title{
A Comprehensive Survey on Agricultural Image Processing
}

\author{
Vivek Ugale ${ }^{1}$, Deepak Gupta ${ }^{2}$ \\ ${ }^{1}$ PG Student, Siddhant College of Engineering, Savitribai Phule Pune University, Pune, \\ ${ }^{2}$ Professor, Siddhant College of Engineering, Savitribai Phule Pune University, Pune
}

\begin{abstract}
Agriculture is important in human life. Human being is directly dependent on agriculture for food requirement. As human population is rapidly increasing by exponential speed, we required to increase the productivity of agriculture crop yield. But now productivity of agriculture crop yield is reducing because of reasons like various kinds of disease, mineral deficiency, etc. Quality of food is also decreases. For human health, productivity as well as quality of agricultural crop must be improve by using new, advanced current computer technologies like robotics, computer vision. Current manual methods of agriculture are very harder, expensive with lowest efficiency due to human limitations. Manually monitoring agriculture field is very time consuming and required more and skilled human resource. Hence current research computer science is going on utilization of advanced computer vision techniques for precision agriculture. Because of computer methods like advanced image processing and computer vision techniques used in agriculture, various decisions are generated by computer system which lead to proper utilization of human recourses and decisions are quicker with increased accuracy. For agriculture image processing, advanced image processing methods and computer vision techniques are grouped according to specific objective like image acquisition, pre-processing, image segmentation, feature extraction and classification. This paper presents survey on advanced image processing methods and computer vision techniques that used for agricultural image processing.
\end{abstract}

Keywords: Computer vision, Precision agriculture, Image segmentation, Feature extraction, Classification.

\section{Introduction}

Food is basic and principal requirement of human, so food has highest precedence in human life. Mostly food is generated from yield of agricultural crops. India as well as many developing countries are cultivated countries and most of population is directly depends on agriculture. Researcher attracts towards agriculture field to increase quantity and quality of agricultural crop yield to feed growing populations. Farmers produces yield from agricultural field. But while farming, farmer face not only environmental problems like storm, heavy rain, draught as well as manmade problems like delay in expert advice of disease, nutrients deficiency problem, limited resources like water, electricity, increasing cost of fertilizers. These problems directly affect on productivity of crop yield and result in most population does not get efficient food to eat and indirectly affect growth as well as wealth of country. So computer science researchers attract towards agriculture field to increase accuracy of automation in robotic technology. For reducing manual labor work, computer technologies pay important role automation of robotics. This paper provides survey about various methods used for agricultural image processing problem solving.

\subsection{Agricultural image processing}

In agricultural image processing, digital image of agriculture crop is used as input for information extraction from which various decisions can be generated. Various diseases and deficiencies of agriculture crop easily detected by just analyzing digital image of infected part like leaves of crop.

\subsection{Precision Agriculture}

In agriculture, many resources are used like human-power, water, electricity, fertilizers. These resources are limited and higher in cost so proper management and utilization required of these resources. Precision agriculture deals with appropriate management of all resources. Due to efficient utilization of resources, quality as well as productivity of agriculture crop yield increases.

\subsection{Image Segmentation}

Pre-processed image is provided as a input to image segmentation step. In image segmentation, pre-processed image is converted into segments according to similarity. For efficient feature extraction, image segmentation step is very important.

\subsection{Feature Extraction and Classification}

In feature extraction step, various attributes of segmented image are extracted. These extracted features can be following type like color feature, texture feature. These features are stored in feature file of that particular image into image knowledge database. Feature file is provided to classifier for classification. According to classification algorithm and feature file, classifier classify input image into predefined categories. Classification process is useful for early detection of disease, identifying nutrient deficiency.

\section{Literature Survey}

In this section, various surveys of computer vision technologies for agricultural image processing are discussed 


\section{International Journal of Science and Research (IJSR) \\ ISSN (Online): 2319-7064}

Index Copernicus Value (2013): 6.14 | Impact Factor (2014): 5.611

and arranged according to their objective to their objectives such as agricultural image processing problem analysis, image acquisition, preprocessing, segmentation methods, feature extraction and classification.

Massimo Minervini et al, in their paper [1], they gave the actual computer vision and image processing challenges in agricultural image processing problem. They also discussed that the main bottleneck was in software i.e. automated analysis algorithm which can be used for analysis of agricultural crop digital image. Algorithm should extract accurate and more precise information from digital images taken from agricultural field of crop in real time. These algorithms should be coping with restrictions of hardware to manage implementation cost. They also discussed that for agricultural image processing problem, computer vision techniques got growing interest from scientifically as well as commercially side because agriculture field have very strong social impact. As computer vision methods extracts useful information from digital image efficiently and accurately, extracted information could used for automated robotic applications for decision making in real time application very effectively.

Aryan Chaudhary et al [2], discussed in their paper that automatic integrated robotic system which uses computer vision techniques for $3 \mathrm{D}$ agricultural plant growth measurement. They suggest that this automatic integrated robotic system capable for capturing 3D plant data in real time and this system would be helpful for large scale crop monitoring; this will reduce huge manual work required for crop inspection over large geographical area.

Khirade et al, in their paper [3], they explained that how agricultural plant disease are detected using image processing techniques. They also discussed classification methods like neural network, back propagation, support vector machine.

They proposed that leaf of crop must get major attention in disease detection process because most disease symptoms shown by leaves of agricultural crop.

Manuel Grand-Brochier et al [5], compared thirteen segmentation methods and concluded after testing that Guided active contour segmentation approach have higher the accuracy than other segmentation method approaches in leaf object identification in natural image. But active guided contour have drawback of under-segmentation. To overcome this under-segmentation drawback, they found solution as to use two preprocessing techniques namely color distance map and input stroke. Finally after all experimental comparisons with various segmentation approaches along with preprocessing methods, they gave comparative study of segmentation methods to tree leaves extraction method.

Gou-quan jiang et al [6] concluded in their study that for segmentation of agricultural image processing application, we must use segmentation based on color feature to increase accuracy. They also stated that RGB image transformation into excess green 2G-R-B index gives advantage in segmentation.
Vijay Singh et al [7] proposed a method which uses image processing technique and genetic algorithm to detect unhealthy region of plant leaves. After performing all experiments in MATLAB [8], authors said that if segmentation using genetic algorithm required very less effort. Segmentation using genetic algorithm, this approach gives advantage to increase the accuracy of recognition rate in classification process.

In the paper [9], Ratnasari et al proposed a new approach that uses segmentation based on threshold and support vector machine as classifier in classification technique for detecting leaf disease on sugarcane. After testing and analyzing the results of proposed approach, they committed that given approach is very useful for early disease identification to reduce the loss in yield of sugarcane. Authors of this paper used support vector machine as classifier for classification of segmented image. Two types of feature were chosen by authors of this paper for feature extraction. Authors of this paper used color feature type and texture feature type. For color feature extraction, they used $\mathrm{L}^{*} \mathrm{a} * \mathrm{~b}$ color space and for texture feature extraction Gray level co-occurrence matrix (GLCM) is used. Authors of this paper said that this proposed model identify sugarcane leaf disease with $80 \%$ accuracy.

Rastogi et al [10] presented scenario of image processing and computer vision in agriculture field as innovative and important problem solving techniques since these techniques are more accurate and quicker than manual methods. For solving agricultural image processing problems in efficient and effective way authors of this paper also suggested to use artificial neural network for agricultural plant leaf disease recognition and classification. After disease classification, grading of disease is also done by calculating amount of infected area of leaf because of disease by using fuzzy logic. Authors of this paper proposed a technique for automatic leaf identification system for precision agriculture using computer vision techniques. Computer vision technologies used to generate useful and required information from digital images of agriculture crop. After information extracted from digital images of agricultural plant leaf, this information is passed to a classified for classification. Authors of this paper used artificial neural network classifier for classification. Artificial neural network classifier classifies information extracted from digital image of infected leaf according to disease type accurately. This helps to recognize disease. This approach is also very useful to grade disease according to risk. Finally authors of this paper discussed in conclusion that this automatic approach to recognize disease definitely reduce manual work and this system very useful to agriculture experts to analyze infected leaf by only naked eye.

Barbebo discussed in their paper [11], various methods of classification methods used for image analysis and disease recognition in agriculture field applications along with the limitations of each approach. 


\section{International Journal of Science and Research (IJSR) \\ ISSN (Online): 2319-7064}

Index Copernicus Value (2013): 6.14 | Impact Factor (2014): 5.611

\section{Conclusion}

This literature survey provides useful guidelines to researchers and various approaches to proceed in agricultural image processing problem. Computer vision techniques are very useful to analyze digital image of agricultural crop. Essential information is extracted from digital image with the help of computer vision techniques. This extracted information is used for various decisions making. This agricultural image processing methods are able to give enhanced output and it has capability of overcoming the human limitations. This improvises utilization of computer techniques to increase agricultural crop yield. Precision agriculture is easily achievable with use of computer vision techniques for decision making. In agricultural image processing, use of smart phone for image acquisition as well as analysis purpose reduces computational equipment cost. Extracted color features provide important information which used classification.

\section{References}

[1] Massimo Minervini, Hanno Scharr, Sotirios A. Tsaftaris, "Image Analysis: The New Bottleneck in Plant Phenotyping" IEEE Signal Magazine, pp.126-131, July 2015

[2] Ayan Chaudhury, Christopher Ward, Ali Talasaz, Alexander G. Ivanov, Norman P.A. Huner, Bernard Grodzinski, Rajni V. Patel, John L. Barron, "Computer Vision Based Autonomous Robotic System for 3D Plant Growth Measurement," In Proceedings of the IEEE $201512^{\text {th }}$ Conference on Computer Robot Vision (CRV), pp. 290-296, 2015.

[3] Sachin D. Khirade and A. B. Patil, "Plant Disease Detection using Image Processing," In Proceedings of the IEEE 2015 International Conference on Computing Communication Control and Automation, pp. 768-771, 2015.

[4] Marion Neumann, Lisa Hallau, Benjamin Klatt, Kristian Kersting, Christian Bauckhage, "Erosion Band Features for Cell Phone Image Based Plant Disease Classification," In Proceedings of the IEEE 2014 International Conference on Pattern Recognition, pp. 3315-3320, 2014

[5] Manuel Grand-brouchier, Antonie Vacavant, Guillaume Cerutti, Camille Kurtz, Jonathan Weber, Laure Tougne, "Tree Leaves Extraction in Natural Images: Comparative Study of Pre-processing Tools and Segmentation Methods," IEEE transaction on Image Processing, 2015.

[6] Guo-quan Jiang, Cui-jun Zhao, Jun-yan Qi, "The Research of Image Segmentation Based on Color Characteristic," In Proceedings of the IEEE 2011 International Conference on Machine Learning and Cybernetics, pp. 1851-1855, 2011.

[7] Vijay Singh, Varsha, A. K. Mishra, "Detection of Unhealthy Region of Plant Leaves Using Image Processing and Genetic Algorithm," In Proceedings of the IEEE 2015 International Conference on Advances in Computer Engineering and Application (ICACEA), pp. 1028-1032, 2015.
[8] MATLAB version 7.14.0 The MathWorks Inc., Feb 2012

[9] Evy Kamilah Ratnasari, Mustika Mentari, Ratih Kartika Dewi, R. V. Hari Ginardi, "Sugarcane Leaf Disease Detection and Severity Estimation Based on Segmented Spots Image," In Proceedings of the IEEE 2014 International Conference on Information Communication Technology and System (ICTS), pp. 93-98, 2014.

[10] Aakanksha Rastogi, Ritika Arora, Shanu Sharma, "Leaf Disease Detection and Grading using Computer Vision Technology and Fuzzy Logic," In Proceedings of the IEEE $20152^{\text {nd }}$ International Conference on Signal Processing and Integrated Networks (SPIN), pp. 500$505,2015$.

[11] Jayme Garcia Arnal Barbebo, "Digital Image Processing Techniques for Detecting, Quantifying and Classifying Plant Disease," SpringerPlus, 2013. 